\title{
THE STRUCTURE OF POINTWISE RECURRENT MAPS HAVING THE PSEUDO ORBIT TRACING PROPERTY
}

\author{
JIEHUA MAI AND XIANGDONG YE
}

\begin{abstract}
We show that a continuous map of a metric space is pointwise recurrent and has the pseudo orbit tracing property if and only if the map is uniformly conjugate to an adding-machine-like map restricted to some invariant subset.
\end{abstract}

\section{$\S 1$. Introduction}

The pseudo orbit tracing property (abbrev. POTP) comes from the study of Anosov diffeomorphisms: POTP and expansiveness imply the stability of the system, see $[\mathrm{AH}]$. POTP has been studied by many authors. For a manifold $M$, this property is generic in the space of all homeomorphisms of $M$ with $C^{0}$-topology [PP]. For a closed interval $I$, in $[\mathrm{K}]$ the author determines all zero entropy continuous maps of $I$ having POTP. For a positive entropy continuous map of $I$ the situation is more complicated. In fact, for the family of tent maps, almost all maps have POTP and the set of parameters for which the maps do not have POTP is locally uncountable $[\mathrm{CKY}]$.

Recurrence is one of the most important subjects in the study of dynamical systems. It is known that a recurrent homeomorphism of the plane or of a compact surface with negative Euler characteristic is periodic ([OT] and $[\mathrm{KP}])$, i.e. some iterates of the homeomorphims are identity. Moreover, a transitive, non-minimal recurrent homeomorphism of a compact metric space does exist $[\mathrm{KW}]$.

In this note we shall discuss the structure of pointwise recurrent maps with POTP. In $[\mathrm{M}]$, the first author of this note studied some properties of pointwise recurrent maps having POTP. In particular, he proved that

Received November 13, 2000.

2000 Mathematics Subject Classification: 54H20, 37B10, 37B20, 37C15.

Both authors are supported by 973 project and NSFC 19625103. 
such maps should be pointwise minimal. Here we completely determine the structure of pointwise recurrent maps having POTP using inverse limit description. To be more precise, we introduce the following notion.

Assume that $X$ is a metric space with metric $d$ and $f: X \rightarrow X$ is a continuous map. For $x \in X$, the orbit of $x$, denoted by $O(x, f)$, is the set $\left\{x, f(x), f^{2}(x), \ldots\right\} . \quad x \in X$ is a recurrent point of $f$, if for each $\epsilon>0$, there is $n=n(\epsilon) \in \mathbb{N}$ such that $d\left(f^{n}(x), x\right)<\epsilon$. The set of periodic points and recurrent points of $f$ are denoted by $P(f)$ and $R(f)$ respectively. $f$ is said to be pointwise recurrent (resp. pointwise periodic) if $R(f)=X$ (resp. $P(f)=X$ ) and $f$ is recurrent if there is $n_{i} \rightarrow+\infty$ such that $\sup _{x \in X} d\left(f^{n_{i}}(x), x\right) \rightarrow 0$ (in some papers it is called uniformly rigid). For a subset $X_{0}$ of $X$, if $f\left(X_{0}\right) \subset X_{0}$, then we say that $X_{0}$ is invariant (under $f$ ) and $f \mid X_{0}: X_{0} \rightarrow X_{0}$ is a subsystem. If $X_{0}$ is a non-empty, closed, invariant subset of $X$ and each non-empty closed proper subset of $X_{0}$ is not invariant, then $X_{0}$ is called a minimal set of $f$. If $X$ is a minimal set of $f$, then $f$ is called a minimal map. It is clear that a minimal map on a space with finitely many points is a cyclic permutation.

Suppose that $\left(x_{n}\right)_{n=0}^{b}$ is a sequence in $X$ with $b \leq+\infty$ and $\delta>0$. If for each $0 \leq n<b$ we have $d\left(x_{n+1}, f\left(x_{n}\right)\right)<\delta$, then $\left(x_{n}\right)_{n=0}^{b}$ is said to be a $\delta$-pseudo orbit of length $b$ (from $x_{0}$ to $x_{b}$ if $b$ is finite). For a given $\epsilon>0$, if there is $y \in X$ such that $d\left(x_{0}, y\right) \leq \epsilon$ and $d\left(x_{n+1}, f^{n+1}(y)\right) \leq \epsilon$ for $0 \leq n<b$, then we say that the $\delta$-pseudo orbit $\left(x_{n}\right)_{n=0}^{b}$ is $\epsilon$-traced by $y$ or the orbit of $y .(X, f)$ is said to have the pseudo orbit tracing property (abbrev. POTP) if for each $\epsilon>0$, there is $\delta=\delta(\epsilon)>0$ such that each $\delta$-pseudo orbit of infinite length can be $\epsilon$-traced by some point in $X$.

Given $K=\left(k_{1}, k_{2}, \ldots\right)$ with $k_{i} \geq 1$, we define $\Sigma_{K}=\prod_{i=1}^{\infty}\left\{0, \ldots, k_{i}-\right.$ $1\}$, where $\left\{0, \ldots, k_{i}-1\right\}$ and $\Sigma_{K}$ are equipped with the discrete and the product topology respectively. If $x=\left(x_{1}, x_{2}, \ldots\right)$ and $y=\left(y_{1}, y_{2}, \ldots\right)$ are two elements of $\Sigma_{K}$ then their sum $x \oplus y=\left(z_{1}, z_{2}, \ldots\right)$ is defined as follows. If $x_{1}+y_{1}<k_{1}$, then $z_{1}=x_{1}+y_{1}$; if $x_{1}+y_{1} \geq k_{1}$, then $z_{1}=x_{1}+y_{1}-k_{1}$ and we carry 1 to the next position. The other terms $z_{2}, \ldots$ are successively determined in the same fashion. Let $f_{K}: \Sigma_{K} \rightarrow \Sigma_{K}$ be defined by $f_{K}(z)=z \oplus 1$ for each $z \in \Sigma_{K}$, where $1=(1,0,0, \ldots)$. It is known that $f_{K}$ is a minimal map, which is called an adding machine (abbrev. AM). We note that if $\left\{i \in \mathbb{N}: k_{i}>1\right\}$ is finite, then $f_{K}$ is periodic and $\Sigma_{K}$ is the unique periodic orbit of $f_{K}$.

Assume that $\psi_{i}: Y_{i} \rightarrow Y_{i}$ is continuous, $Y_{i}$ is a metric space, $i=1,2$. 
$\psi_{1}$ is said to be conjugate to $\psi_{2}$ if there is a homeomorphism $h: Y_{1} \rightarrow Y_{2}$ such that $h \psi_{1}=\psi_{2} h$. If both $h$ and $h^{-1}$ are uniformly continuous, then we say that $\psi_{1}$ is uniformly conjugate to $\psi_{2}$.

Let $\left(X_{i}, d_{i}\right)$ be a metric space and $\phi_{i}: X_{i+1} \rightarrow X_{i}$ be continuous onto maps for $i \in \mathbb{N}$. Define $X_{\infty}=\operatorname{inv} \lim \left\{X_{i}, \phi_{i}\right\}=\left\{\left(x_{1}, x_{2}, \ldots\right): x_{i} \in X_{i}\right.$, $\left.\phi_{i}\left(x_{i+1}\right)=x_{i}, i \in \mathbb{N}\right\} . \quad X_{\infty}$ is called the inverse limit space of $\left\{X_{i}, \phi_{i}\right\}$ and $\phi_{i}$ is called the bonding map. As a subspace of the product space $\prod_{i=1}^{\infty} X_{i}, X_{\infty}$ is a metric space with a compatible metric $d_{\infty}$ defined by $d_{\infty}(x, y)=\sum_{i=1}^{\infty} d_{i}\left(x_{i}, y_{i}\right) /\left(2^{i}\left(1+d_{i}\left(x_{i}, y_{i}\right)\right)\right)$ for $x=\left(x_{1}, x_{2}, \ldots\right), y=$ $\left(y_{1}, y_{2}, \ldots\right) \in X_{\infty}$.

If $f_{i}: X_{i} \rightarrow X_{i}$ is continuous and satisfies that $\phi_{i} f_{i+1}=f_{i} \phi_{i}$ for $i \in \mathbb{N}$, then $\left\{f_{i}\right\}$ induces a map $f_{\infty}$ on $X_{\infty}$ defined by $f_{\infty}\left(x_{1}, x_{2}, \ldots\right)=$ $\left(f_{1}\left(x_{1}\right), f_{2}\left(x_{2}\right), \ldots\right)$. We refer $[\mathrm{N}]$ for the basic properties of the inverse limit space and the induced map. If for each $i \in \mathbb{N},\left(X_{i}, d_{i}\right)$ is a standard discrete metric space, i.e. $d_{i}(x, y)=1$ for all $x \neq y \in X_{i}$, and $f_{i}$ is pointwise periodic, then $f_{\infty}$ is called an AM-like map. Obviously, every AM is topologically conjugate to an AM-like map (in this case each $f_{i}$ has only one periodic orbit). It is easy to see if $W$ is a non-empty invariant subset of an AM-like map, then $f_{\infty} \mid W: W \rightarrow W$ has POTP.

Now we are ready to state the main result of the paper.

Main Theorem. Let $X$ be a metric space and $f: X \rightarrow X$ be continuous. Then

(1) $f$ is pointwise recurrent and has POTP if and only if $f$ is uniformly conjugate to a subsystem of some AM-like map.

(2) If $X$ is complete, then $f$ is pointwise recurrent and has POTP if and only if $f$ is uniformly conjugate to some AM-like map.

(3) $f$ is a minimal map and has POTP if and only if $f$ is uniformly conjugate to a subsystem of some AM map.

In $[\mathrm{AH}]$ the authors show that minimal homeomorphisms of connected compact metric spaces having more than one point do not have POTP. Theorem A generalizes the result. By [A] distal homeomorphisms of connected compact metric spaces having more than one point do not have POTP. Theorem A also generalizes the result as distal homeomorphisms are pointwise recurrent. We remark that if we replace pointwise recurrent by pointwise non-wandering, the above theorem is false, the full shift serves as such an example. 


\section{§2. Some properties of pointwise recurrent maps having POTP}

Assume that $(X, d)$ is a metric space and $f: X \rightarrow X$ is continuous. A partition of $X$ is a family of disjoint non-empty subsets of $X$ whose union is all of $X$. Suppose $\epsilon$ and $\delta$ are positive numbers. A partition $\left\{X_{\alpha}: \alpha \in A\right\}$ (where $A$ is an index set) is called a $\delta$-decomposition of $X$ if $d\left(X_{\alpha}, X_{\beta}\right) \geq \delta$ for all $\alpha$ and $\beta \in A$ with $\alpha \neq \beta$, and is said to be $f$-invariant if $f\left(X_{\alpha}\right) \subset X_{\alpha}$ for all $\alpha \in A$. An $\delta$-decomposition $\left\{X_{\alpha}: \alpha \in A\right\}$ is called an $(\epsilon, \delta)$-decomposition if $\operatorname{diam}\left(X_{\alpha}\right) \leq \epsilon$ for all $\alpha \in A$. It is obvious that if $\left\{X_{\alpha}: \alpha \in A\right\}$ is an $\delta$-decomposition, then each $X_{\alpha}$ is open and closed.

For $\delta>0$ define a relation $\sim_{\delta, f}$ on $X$ as follows: $x \sim_{\delta, f} y$ if and only if there exist $x_{0}, \ldots, x_{k} \in X$ such that $x=x_{0}, y=x_{k}$ and $d\left(O\left(x_{i-1}, f\right)\right.$, $\left.O\left(x_{i}, f\right)\right)<\delta$ for each $i \in \mathbb{N}_{k}$, where $\mathbb{N}_{k}=\{1, \ldots, k\}$. It is easy to verify that $\sim_{\delta, f}$ is an equivalence relation on $X$. This relation induces a partition $\left\{X_{\alpha \delta}: \alpha \in A(\delta)\right\}$, where $A(\delta)$ is an index set depending on $\delta$. It is easy to see

LEMmA 2.1. The partition $\left\{X_{\alpha \delta}: \alpha \in A(\delta)\right\}$ induced by $\sim_{\delta, f}$ is an $f$-invariant $\delta$-decomposition of $X$.

In the sequel we assume that $f: X \rightarrow X$ is pointwise recurrent.

Lemma 2.2. For $x, y \in X, x \sim_{\delta, f} y$ if and only if there exists $a \delta$ pseudo orbit of finite length from $x$ to $y$.

For $\alpha \in A(\delta)$ and $x, y \in X_{\alpha \delta}$, let

$$
\begin{aligned}
& Z(x, y, \delta, f)= \\
& \quad\left\{i \in \mathbb{Z}_{+}: \text {there is a } \delta \text {-pseudo orbit of length } i \text { from } x \text { to } y\right\}
\end{aligned}
$$

For convenience, we regard $(x)$ as a $\delta$-pseudo orbit from $x$ to $x$ of length 0 . Thus, $0 \in Z(x, y, \delta, f)$ if and only if $x=y$.

For two nonempty subsets $M_{1}$ and $M_{2}$ of $\mathbb{Z}$, denote $\left\{i+j: i \in M_{1}, j \in\right.$ $\left.M_{2}\right\}$ and $\left\{-i: i \in M_{1}\right\}$ by $M_{1}+M_{2}$ and $-M_{1}$ respectively. Then we have

$$
Z(x, y, \delta, f)+Z(y, x, \delta, f) \subset Z(x, x, \delta, f) \cap Z(y, y, \delta, f)
$$

and

$$
Z(x, y, \delta, f)+Z(y, y, \delta, f)+Z(y, x, \delta, f) \subset Z(x, x, \delta, f)
$$


From (2.2) and (2.3) we get

$$
\begin{aligned}
Z(y, y, \delta, f) \subset & (-Z(x, y, \delta, f))+Z(x, y, \delta, f)+Z(y, y, \delta, f) \\
& +Z(y, x, \delta, f)+(-Z(y, x, \delta, f)) \\
\subset & Z(x, x, \delta, f)+(-Z(x, y, \delta, f))+(-Z(y, x, \delta, f)) \\
\subset & Z(x, x, \delta, f)+(-Z(x, x, \delta, f)) .
\end{aligned}
$$

For a subset $M \subset \mathbb{Z}$, let $\operatorname{gcd}(M)$ denote the greatest common divisor of numbers in $M$. It is easy to see that $\operatorname{gcd}(M+(-M))=\operatorname{gcd}(M)$, and if $M^{\prime} \subset M$ then $\operatorname{gcd}(M)$ is a divisor of $\operatorname{gcd}\left(M^{\prime}\right)$. Thus, it follows from (2.4) that $\operatorname{gcd}(Z(x, x, \delta, f))$ is a divisor of $\operatorname{gcd}(Z(y, y, \delta, f))$. By the same reasoning $\operatorname{gcd}(Z(y, y, \delta, f))$ is a divisor of $\operatorname{gcd}(Z(x, x, \delta, f))$. Thus, we have

$$
\operatorname{gcd}(Z(x, x, \delta, f))=\operatorname{gcd}(Z(y, y, \delta, f)), \quad \text { for all } x, y \in X_{\alpha \delta} \text {. }
$$

Now fix $v \in X_{\alpha \delta}$ and set

$$
n_{\alpha \delta}=n(\alpha, \delta)=n(\alpha, \delta, f)=\operatorname{gcd}(Z(v, v, \delta, f)) .
$$

Lemma 2.3. For any $x, y \in X_{\alpha \delta}$ and $i, j \in Z(x, y, \delta, f)$, we have $n_{\alpha \delta} \mid(i-j)$.

Proof. Take $b \in Z(y, x, \delta, f)$. By (2.2) we have $\{i+b, j+b\} \subset$ $Z(x, x, \delta, f)$. By $(2.5)$ and $(2.6)$, it follows that $\left\{(i+b) / n_{\alpha \delta},(j+b) / n_{\alpha \delta}\right\} \subset \mathbb{Z}$. Thus, $n_{\alpha \delta} \mid(i-j)$.

For $n, j \in \mathbb{Z}$ write $n \mathbb{Z}+j=\{n i+j: i \in \mathbb{Z}\}$. Then by Lemma 2.3 we have

Lemma 2.4. For $x, y \in X_{\alpha \delta}$ there is a unique $\mu(x, y) \in\left\{0,1, \ldots, n_{\alpha \delta}-\right.$ $1\}$ such that $Z(x, y, \delta, f) \subset n_{\alpha \delta} \mathbb{Z}+\mu(x, y)$.

It is easy to verify that

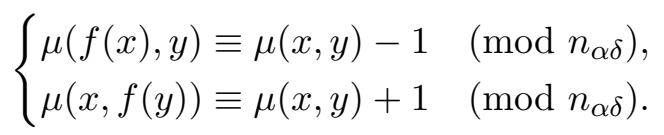

For $i=0,1, \ldots, n_{\alpha \delta}-1$ set

$$
W_{i}=W_{i \alpha \delta}=\left\{x \in X_{\alpha \delta}: \mu(v, x)=i\right\} .
$$

It is easy to see that $\left\{W_{0}, \ldots, W_{n_{\alpha \delta}-1}\right\}$ is a partition of $X_{\alpha \delta}$. Moreover, from (2.7), we have

$$
f\left(W_{n_{\alpha \delta}-1}\right) \subset W_{0}, \text { and } f\left(W_{i}\right)=W_{i+1} \text { for } 0 \leq i \leq n_{\alpha \delta}-2 .
$$


LEMMA 2.5. There exists $b \in \mathbb{Z}_{+}$such that $n_{\alpha \delta} \mathbb{Z} \cap[b, \infty) \subset Z(v, v, \delta, f)$.

Proof. From (2.6) there exists $\left\{m_{1}, \ldots, m_{k}\right\} \subset Z(v, v, \delta, f) \backslash\{0\}$ such that $\operatorname{gcd}\left(m_{1}, \ldots, m_{k}\right)=n_{\alpha \delta}$. Set $m_{0}=\min (Z(v, v, \delta, f) \backslash\{0\})$ and $\mu_{i}=$ $m_{i} / n_{\alpha \delta}$ for $0 \leq i \leq k$. Then $\operatorname{gcd}\left(\mu_{1}, \ldots, \mu_{k}\right)=1$. Thus, there exists $\left\{\lambda_{1}, \ldots, \lambda_{k}\right\} \subset \mathbb{Z}$ with $\sum_{i=1}^{k} \lambda_{i} \mu_{i}=1$. This implies that $\sum_{i=1}^{k} \lambda_{i} m_{i}=n_{\alpha \delta}$. Let $b_{1}=\sum_{i=1}^{k}\left|\lambda_{i} m_{i}\right|$ and $b_{2}=\mu_{0} b_{1}$. From (2.2) we know that for $j=$ $0,1, \ldots, \mu_{0}$,

$$
b_{2}-j n_{\alpha \delta}=\sum_{i=1}^{k}\left(\mu_{0}\left|\lambda_{i}\right|-j \lambda_{i}\right) m_{i} \in Z(v, v, \delta, f) .
$$

Noting $m_{0}=\mu_{0} n_{\alpha \delta} \in Z(v, v, \delta, f)$, by (2.10) and (2.2) we have for each $j \in \mathbb{N}, b_{2}+j n_{\alpha \delta} \in Z(v, v, \delta, f)$. Thus, $[b, \infty) \cap n_{\alpha \delta} \mathbb{Z} \subset Z(v, v, \delta, f)$ if we set $b=b_{2}-m_{0}$.

Assume that $L=\left(x_{0}, \ldots, x_{k}\right)$ and $L_{1}=\left(y_{0}, \ldots, y_{m}\right)$ are two finite $\delta$ pseudo orbits of $f$, and $L_{2}=\left(z_{0}, z_{1}, \ldots\right)$ is a $\delta$-pseudo orbit of $f$. If $x_{k}=y_{0}$ denote $\left(x_{0}, \ldots, x_{k}, y_{1}, \ldots, y_{m}\right)$ by $L L_{1}$; if $x_{k}=z_{0}$, denote $\left(x_{0}, \ldots, x_{k}, z_{1}\right.$, $\left.z_{2}, \ldots\right)$ by $L L_{2}$, if $x_{k}=x_{0}$ and $k>0$, denote $L \cdots L(j$ times $)$ by $L^{j}$, denote $L L \cdots$ by $L^{\infty}$.

Lemma 2.6. Suppose $x \in W_{i}, 0 \leq i \leq n_{\alpha \delta}-1$ and $y \in X_{\alpha \delta}$. If $d(x, y)<\delta$, then $y \in W_{i}$.

Proof. Let $L$ be a $\delta$-pseudo orbit from $v$ to $x$ of length $m$. Then $m \equiv i$ $\left(\bmod n_{\alpha \delta}\right)$. As $x$ is a recurrent point of $f, x$ is also a recurrent point of $f^{n_{\alpha \delta}}$. Thus, there exists $k \in \mathbb{N}$ such that $d\left(f^{k n_{\alpha \delta}}(x), x\right)<\delta-d(x, y)$. Set

$$
L^{\prime}=\left(L, f(x), f^{2}(x), \ldots, f^{k n_{\alpha \delta}-1}(x), y\right) .
$$

Then $L^{\prime}$ is a $\delta$-pseudo orbit from $v$ to $y$ of length $m+k n_{\alpha \delta}$. As $m+k n_{\alpha \delta} \equiv$ $m \equiv i\left(\bmod n_{\alpha \delta}\right), y \in W_{i}$.

A sequence $\left(W_{0}, \ldots, W_{k}\right)$ of subsets of $X$ is said to be $f$-cyclic if $f\left(W_{k}\right) \subset W_{0}$ and $f\left(W_{i-1}\right) \subset W_{i}$ for $i=1, \ldots, k$.

By Lemma 2.6 and (2.9) we get immediately

LEMMA 2.7. $\left(W_{0 \alpha \delta}, W_{1 \alpha \delta}, \ldots, W_{\left(n_{\alpha \delta}-1\right) \alpha \delta}\right)$ is a $f$-cyclic $\delta$-decomposition of $X_{\alpha \delta}$. 
From now on we assume that $f$ is pointwise recurrent and has POTP. Then, there is a function $\eta:(0, \infty) \rightarrow(0, \infty)$ such that for each $\epsilon>0$, each $\eta(\epsilon)$-pseudo orbit of $f$ can be $\epsilon / 4$-traced by some orbit of $f$ and $\eta(\epsilon) \leq \epsilon$. For any given $\epsilon>0$, set $\delta=\eta(\epsilon)$.

LEMMA 2.8. For each $0 \leq i \leq n_{\alpha \delta}-1$ we have $\operatorname{diam}\left(W_{i \alpha \delta}\right) \leq \epsilon$.

Proof. Let $x, y \in W_{i \alpha \delta}$. Assume that $L_{1}$ is a $\delta$-pseudo orbit from $x$ to $v, L_{3}$ is a $\delta$-pseudo orbit from $v$ to $y$ and $L_{4}$ is a $\delta$-pseudo orbit from $y$ to $v$. Suppose the length of $L_{j}$ is $c_{j}$ for $j=1,3,4$. By (2.8) and (2.6), we get $c_{3} \equiv i\left(\bmod n_{\alpha \delta}\right), c_{3}+c_{4} \equiv 0\left(\bmod n_{\alpha \delta}\right)$ and $c_{1}+c_{3} \equiv 0\left(\bmod n_{\alpha \delta}\right)$. By Lemma 2.5, there is a $\delta$-pseudo orbit $L_{2}$ from $v$ to $v$ of length $c_{2}$ such that $c_{1}+c_{2}+c_{3} \equiv 0\left(\bmod m_{0}\right)$, where $m_{0}=\min (Z(v, v, \delta, f) \backslash\{0\})$ as above.

Let $L_{5}=\left(v_{0}, \ldots, v_{m_{0}}\right)$ be a $\delta$-pseudo orbit from $v$ to $v$ of length $m_{0}$. Set $L=L_{1} L_{2} L_{3} L_{4} L_{5}^{\infty}$. Then $L$ is a $\delta$-pseudo orbit of $f$. As $\delta=\eta(\epsilon), L$ can be $\epsilon / 4$-traced by some point $w \in X$. Let $w_{j}=f^{j}(w), c=c_{1}+c_{2}+c_{3}+c_{4}$ and $Y_{j}=O\left(w_{c+j}, f^{m_{0}}\right),\left(j \in \mathbb{Z}_{+}\right)$. As $w$ is a recurrent point of $f$, we have

$$
O(w, f) \subset \overline{O\left(w_{c}, f\right)}=\bigcup_{k=0}^{m_{0}-1} \overline{Y_{k}}
$$

and

$$
f\left(Y_{j}\right)=Y_{j+1}, \text { and } \overline{Y_{j+m_{0}}} \subset \overline{Y_{j}}, j \in \mathbb{Z}_{+} .
$$

From (2.11), there exists $q \in\left\{0,1, \ldots, m_{0}-1\right\}$ such that $w \in \overline{Y_{q}}$. Let $a=c_{1}+c_{2}+c_{3}$. As $a \in m_{0} \mathbb{Z}$, by $(2.12)$ we have $w_{a} \in \overline{Y_{q}}$. Since $L_{5}^{\infty}$ is $\epsilon / 4$ traced by $O\left(w_{c}, f\right)$, we have $\overline{Y_{k}} \subset \overline{B\left(v_{k}, \epsilon / 4\right)}$ for $0 \leq k<m_{0}$. As $L_{1} L_{2} L_{3}$ is $\epsilon / 4$-traced by $\left(w, f(w), \ldots, f^{a}(w)\right)$, we have $d\left(x, \overline{Y_{q}}\right) \leq d(x, w)<\epsilon / 4$ and $d\left(y, \overline{Y_{q}}\right) \leq d\left(y, w_{a}\right)<\epsilon / 4$. Thus,

$$
d(x, y)<\epsilon / 4+\operatorname{diam}\left(\overline{Y_{q}}\right)+\epsilon / 4 \leq \epsilon
$$

for any $x, y \in W_{i \alpha \delta}$. Hence, $\operatorname{diam}\left(W_{i \alpha \delta}\right) \leq \epsilon$.

By Lemmas 2.8 and 2.7 we obtain

LEMMA 2.9. $\left\{W_{i \alpha \delta}: 0 \leq i \leq n_{\alpha \delta}-1\right\}$ is an $(\epsilon, \delta)$-decomposition of $X_{\alpha \delta}$.

Remark 2.10. By Lemmas 2.9 and 2.6, if $\operatorname{diam}\left(X_{\alpha \delta}\right)>\epsilon$ then $n_{\alpha \delta}>1$, and if $\operatorname{diam}\left(X_{\alpha \delta}\right)<\delta$ then $n_{\alpha \delta}=1$. 


\section{§3. Proof of Main Theorem}

Proof of Main Theorem. The sufficiency of the theorem is obvious and it remains to show the necessity of the theorem. Let $\eta:(0, \infty) \rightarrow(0, \infty)$ be the function defined in Section 2. Set $\epsilon_{0}=1$. For $j=0,1, \ldots$, let $\delta_{j}=\eta\left(\epsilon_{j}\right)$ and $\epsilon_{j+1}=\delta_{j} / 2$. Set

$$
S_{j}=\left\{W_{i \alpha \delta_{j}}: i=0,1, \ldots, n_{\alpha \delta_{j}-1}, \text { and } \alpha \in A\left(\delta_{j}\right)\right\} .
$$

Each $W_{i \alpha \delta_{j}}$ can be regarded as a "point" of $S_{j}$. Let $\rho_{j}$ be the standard discrete metric on $S_{j}$. Then $\left(S_{j}, \rho_{j}\right)$ is a standard discrete metric space. Define $\phi_{j}:\left(S_{j+1}, \rho_{j+1}\right) \rightarrow\left(S_{j}, \rho_{j}\right)$ such that

$$
\begin{aligned}
& \phi_{j}\left(W_{k \beta \delta_{j+1}}\right)=W_{i \alpha \delta_{j}} \text { if and only if, } \\
& \text { as subsets of } X, W_{k \beta \delta_{j+1}} \subset W_{i \alpha \delta_{j}} .
\end{aligned}
$$

As $\epsilon_{j+1}<\delta_{j}$, by Lemma 2.9 each point of $S_{j+1}$ (as a subset of $X$ ) is contained in a unique point of $S_{j}$. Thus, $\phi_{j}$ is well defined. It is easy to see that $\phi_{j}$ is surjective and continuous. Hence, we get an inverse limit space

$$
\left(S_{\infty}, \rho_{\infty}\right)=\operatorname{inv} \lim \left\{\left(S_{j}, \rho_{j}\right), \phi_{j}\right\}
$$

Define $g_{j}:\left(S_{j}, \rho_{j}\right) \rightarrow\left(S_{j}, \rho_{j}\right)$ such that for each $\alpha \in A\left(\delta_{j}\right)$

$$
g_{j}\left(W_{i \alpha \delta_{j}}\right)= \begin{cases}W_{0 \alpha \delta_{j}}, & \text { if } i=n_{\alpha \delta_{j}}-1, \\ W_{(i+1) \alpha \delta_{j}}, & \text { if } 0 \leq i \leq n_{\alpha \delta_{j}}-2 .\end{cases}
$$

By (2.9),

$$
g_{j}\left(W_{i \alpha \delta_{j}}\right)=W_{k \beta \delta_{j}} \text { if and only if } f\left(W_{i \alpha \delta_{j}}\right) \subset W_{k \beta \delta_{j}} .
$$

By (3.1) and (3.4) we have $g_{j} \phi_{j}=\phi_{j} g_{j+1}$. Thus, $\left(g_{0}, g_{1}, \ldots\right)$ induces a map $g_{\infty}:\left(S_{\infty}, \rho_{\infty}\right) \rightarrow\left(S_{\infty}, \rho_{\infty}\right)$. By $(3.3), g_{j}$ is pointwise periodic and hence $g_{\infty}$ is an AM-like map.

Each $\sigma=\left(W_{i_{0} \alpha_{0} \delta_{0}}, W_{i_{1} \alpha_{1} \delta_{1}}, \ldots\right) \in S_{\infty}$ is a sequence of subsets of $X$ with $W_{i_{0} \alpha_{0} \delta_{0}} \supset W_{i_{1} \alpha_{1} \delta_{1}} \supset \cdots$. Set $X_{\sigma}=\bigcap_{j=0}^{\infty} W_{i_{j} \alpha_{j} \delta_{j}}$. As $\lim _{j \rightarrow \infty} \epsilon_{j}=0$, by Lemma $2.8 X_{\sigma}$ contains at most one point. Since each $W_{i_{j} \alpha_{j} \delta_{j}}$ is a closed and open subset of $X, X_{\sigma}$ contains exact one point if $X$ is complete. If $X_{\sigma} \neq \emptyset$, set $X_{\sigma}=\left\{x_{\sigma}\right\}$.

As each $S_{j}$ is a partition of $X$, for each $y \in X$, there is a unique $\sigma(y) \in S_{\infty}$ such that $y=x_{\sigma(y)}$. Thus, we may define $h: X \rightarrow S_{\infty}$ by 
$h(y)=\sigma(y)$ for each $y \in X$. As $\lim _{j \rightarrow \infty} \epsilon_{j}=0, h$ is injective. Moreover, if $X$ is complete, $h$ is surjective.

Define $p_{j}: S_{\infty} \rightarrow S_{0} \times S_{1} \times \cdots \times S_{j}$ such that

$$
p_{j}\left(W_{i_{0} \alpha_{0} \delta_{0}}, W_{i_{1} \alpha_{1} \delta_{1}}, W_{i_{2} \alpha_{2} \delta_{2}}, \ldots\right)=\left(W_{i_{0} \alpha_{0} \delta_{0}}, \ldots, W_{i_{j} \alpha_{j} \delta_{j}}\right)
$$

By Lemma 2.9, for $x, y \in X$ with $d(x, y)<\delta_{j}, p_{j} h(x)=p_{j} h(y)$. Thus, $h$ is uniformly continuous. At the same time, for $\sigma, \sigma^{\prime} \in h(X)$, if $p_{j}(\sigma)=p_{j}\left(\sigma^{\prime}\right)$, by Lemma $2.8, d\left(h^{-1}(\sigma), h^{-1}\left(\sigma^{\prime}\right)\right) \leq \epsilon_{j}$. Thus, $h^{-1} \mid h(X)$ is uniformly continuous. Hence $h: X \rightarrow h(X)$ is a uniform homeomorphism.

It is easy to see from (3.4) that $g_{\infty} h=h f$. Hence $f$ is uniformly conjugate to $g_{\infty} \mid h(X)$. This proves (1) and (2) of Theorem A.

If $f$ is minimal, for each $\delta>0, A(\delta)$ is a singleton. Thus for $j \geq 0$, by Lemma 2.7, $g_{j}: S_{j} \rightarrow S_{j}$ is a cyclic permutation. This proves (3) of Theorem A.

\section{REFERENCES}

[A] N. Aoki, Homeomorphisms without the pseudo orbit tracing property, Nagoya Math. J., 88 (1982), 155-160.

$[\mathrm{AH}] \quad$ N. Aoki and K. Hiraide, Topological theory of dynamical systems, Elsevier Science B.V., Netherland, 1994.

[CKY] E. Coven, I. Kan and J. Yorke, Pseudo-orbit shadowing in the family of tent maps, Trans. Amer. Math. Soc., 308 (1988), 227-241.

[K] M. Kuchta, Shadowing property of continuous maps with zero topological entropy, Proc. Amer. Math. Soc., 119 (1993), 641-648.

[KP] B. Kolev and M.-C. Peroueme, Recurrent surface homeomorphism, Math. Proc. Camb. Phil. Soc., 124 (1998), 161-168.

[KW] Y. Katznelson and B. Weiss, When all points are recurrent/generic, Ergodic Theory and Dynamical Systems I (College Park, 1979-80) (A. Katok, ed.), Progr. Math. 10, Birkhauser Verlag, Boston (1981), pp. 195-210.

[M] J. H. Mai, Pointwise recurrent dynamical systems with pseudo orbit tracing property, Northeast Math. J., 12 (1996), 73-78.

[N] S. B. Nadler Jr, Continuum Theory, Pure and Appl. Math. 158, 1992.

[OT] L. G. Oversteegen and E. D. Tymchatyn, Recurrent homeomorphisms on $\mathbb{R}^{2}$ are periodic, Proc. Amer. Math. Soc., 110 (1990), 1083-1087.

[PP] S. Yu. Pilyugin and O. B. Plamenevskaya, Shadowing is generic, Topology Appl., 97 (1999), 253-266. 
Jiehua Mai

Institute of Mathematics

Shantou University

Guangdong, 515063

P. R. China

jhmai@stu.edu.cn

Xiangdong Ye

Department of Mathematics

University of Science and Technology of China

Hefei, Anhui, 230026

P. R. China

yexd@ustc.edu.cn 